\title{
AN INEQUALITY FOR EIGENVALUES OF STURM-LIOUVILLE PROBLEMS
}

\author{
CATHERINE BANDLE AND GÉRARD PHILIPPIN
}

\begin{abstract}
By means of simple transformations, inequalities for eigenvalues corresponding to different boundary conditions are derived.
\end{abstract}

1. Let $\rho(x)>0$ be a continuous function and consider the following eigenvalue problems:

$$
\phi^{\prime \prime}(x)+\lambda \rho(x) \phi(x)=0 \quad \text { in }(-1,1), \quad \phi(-1)=\phi(1)=0,
$$

and

$$
\psi^{\prime \prime}(x)+\mu \rho(x) \psi(x)=0 \quad \text { in }(-1,1), \quad \psi^{\prime}(-1)=\psi^{\prime}(1)=0 .
$$

It is well known that there exist two countable sequences of eigenvalues $0<\lambda_{1}<$ $\lambda_{2}<\cdots$ and $0=\mu_{1}<\mu_{2}<\cdots$ both tending to infinity as $n \rightarrow \infty$. According to Poincaré's principle they may be characterized as

$$
\lambda_{n}=\inf _{L_{n}^{\prime}} \max _{v \in L_{n}^{\prime}} R[v] \quad \text { and } \quad \mu_{n}=\inf _{L_{n}} \max _{v \in L_{n}} R[v]
$$

where

$$
R[v]:=\int_{-1}^{1} v^{\prime 2} d x / \int_{-1}^{1} v^{2} \rho d x
$$

is the Rayleigh quotient, $L_{n}^{\prime} \subset H_{0}^{1}(-1,1)$ and $L_{n} \subset H^{1}(-1,1)$ are $n$-dimensional function spaces. In (1.3), equality holds if and only if $v$ is the $n$th eigenfunction.

2. From (1.3) it follows immediately that

$$
\mu_{n}<\lambda_{n} .
$$

The aim of this note is to sharpen inequality (2.1) for a special class of mass densities $\rho$.

3. Let us start with two elementary lemmas. The first observation is essentially due to $[\mathbf{3}]$.

LEMMA 1. Let $\phi_{1}$ and $\phi_{n}$ be the first and the nth eigenfunctions of (1.1). Then $v_{n}(x)=\phi_{n}(x) / \phi_{1}(x)$ is the nth eigenfunction of

$$
\left\{\phi_{1}^{2} v_{n}^{\prime}\right\}^{\prime}+\tilde{\omega} \phi_{1}^{2} \rho v_{n}=0 \quad \text { in }(-1,1), \quad v_{n}^{\prime}(-1)=v_{n}^{\prime}(1)=0,
$$

with the corresponding eigenvalue $\tilde{\omega}_{n}=\lambda_{n}-\lambda_{1}$.

PROOF. Since $\phi_{k}$ has the expansion $\phi_{k}(x)=\phi_{k}^{\prime}(-1)(x+1)+o\left((x+1)^{2}\right)$, $\phi_{k}^{\prime}(-1) \neq 0$, it follows that

$$
v^{\prime}(x)=\frac{\phi_{n}^{\prime}(x) \phi_{1}(x)-\phi_{n}(x) \phi_{1}^{\prime}(x)}{\phi_{1}^{2}(x)}=O(x+1),
$$

Received by the editors February 5, 1986.

1980 Mathematics Subject Classification (1985 Revision). Primary 34B25. 
which implies $v^{\prime}(-1)=0$. Similarly we have $v^{\prime}(1)=0$. The remaining part is verified by a straightforward computation.

The next result is a slightly modified version of a lemma by Payne and Weinberger [2].

LEMMA 2. Let $v_{n}$ be the $n$th eigenfunction of (3.1). Then $w_{n-1}:=v_{n}^{\prime} \phi_{1}$ is the $(n-1)$ st eigenfunction of

$$
\begin{gathered}
\left(\frac{w^{\prime}}{\rho}\right)^{\prime}-\frac{1}{\rho}\left[\frac{2 \phi_{1}^{\prime 2}}{\phi_{1}^{2}}+\frac{\phi_{1}^{\prime} \rho^{\prime}}{\phi_{1} \rho}\right] w+\nu w=0 \quad \text { in }(-1,1), \\
w(-1)=w(1)=0
\end{gathered}
$$

with the corresponding eigenvalue $\nu_{n-1}=\lambda_{n}-2 \lambda_{1}$.

The proof of (3.2) follows from a straightforward computation. In view of Poincaré's variational principle we have

$$
\nu_{n-1}=\inf _{L_{n-1}^{\prime}} \max _{v \in L_{n-1}^{\prime}}\left\{\int_{-1}^{1} \frac{v^{\prime 2}}{\rho} d x+\int_{-1}^{1}\left[\frac{2 \phi_{1}^{\prime 2}}{\phi_{1}^{2}}+\frac{\phi_{1}^{\prime} \rho^{\prime}}{\phi_{1} \rho}\right] \frac{v^{2}}{\rho} d x\right\} / \int_{-1}^{1} v^{2} d x
$$

where $L_{n-1}^{\prime}$ is an $(n-1)$-dimensional space of functions in $H_{0}^{1}(-1,1)$ for which (3.3) makes sense. If the assumption

$$
\frac{2 \phi_{1}^{\prime 2}}{\phi_{1}^{2}}+\frac{\phi_{1}^{\prime} \rho^{\prime}}{\phi_{1} \rho} \geq 0
$$

holds, then we have by (3.3)

$$
\nu_{n-1} \geq \omega_{n-1},
$$

where $\omega_{n-1}$ is the $(n-1)$ st eigenvalue of

$$
\left(\frac{u^{\prime}}{\rho}\right)^{\prime}+\omega u=0 \quad \text { in }(-1,1), \quad u(-1)=u(1)=0 .
$$

LEMMA 3. $\omega_{n-1}=\mu_{n}$, where $\mu_{n}$ is the nth eigenvalue of (1.2).

Proof. Put $u^{\prime} / \rho=\psi$. Then $\psi^{\prime}=-\omega u$ and $\psi^{\prime \prime}+\omega \rho \psi=0$, which proves the assertion.

We are now ready to establish our main result.

THEOREM 1. Under the assumptions

(i) $\rho(-x)=\rho(x)$, and

(i) $\rho(x)$ increasing in $(-1,0)$, we have $\lambda_{n}-2 \lambda_{1} \geq \mu_{n}$.

PROOF. We first consider the case where $\rho$ is differentiable. In view of assumption (i), $\phi_{1}$ is symmetric with respect to $x=0$. Moreover $\left|\phi_{1}(x)\right|$ is concave, hence $\phi_{1}^{\prime} \rho^{\prime} / \phi_{1} \geq 0$ in $(-1,1)$. Thus assumption (A) is satisfied. The assertion now follows from (3.4) together with Lemmas 2 and 3. If $\rho$ is not differentiable we approximate $\rho$ by means of differentiable functions $\left\{\rho_{n}\right\}_{n=1}^{\infty}$ and use the fact that $\lambda_{n}$ and $\mu_{n}$ depend continuously on $\rho[1$, p. 418]. 
4. Consider the eigenvalue prolem with mixed boundary conditions

$$
\phi^{\prime \prime}(x)+\Lambda \rho(x) \phi(x)=0 \quad \text { in }(0,1), \quad \phi(0)=\phi^{\prime}(1)=0 .
$$

It is easily seen that for the first eigenfunction $\phi_{1}$ we have $\phi_{1} \phi_{1}^{\prime}>0$ in $(0,1)$. If

(iii) $\rho(x)$ is increasing in $(0,1)$,

then condition (A) is satisfied. The same arguments as for Theorem 1 yield

THEOREM 2. Assume (iii). Then $\Lambda_{n}-2 \Lambda_{1} \geq \mu_{n}$.

We finally consider the more general eigenvalue problem

$$
\left(\sigma(x) \phi^{\prime}(x)\right)^{\prime}+\lambda \rho(x) \phi(x)=0 \quad \text { in }(-1,1)
$$

with either boundary conditions

$$
\begin{gathered}
\phi(-1)=\phi(1)=0, \\
\phi(-1)=\phi^{\prime}(1)=0,
\end{gathered}
$$

or

$$
\phi^{\prime}(-1)=\phi^{\prime}(1)=0 .
$$

If we introduce the new variable $t=\int_{-1}^{x}(d s / \sigma(s)),(4.2)$ becomes

$$
\ddot{\phi}+\lambda \sigma(x(t)) \rho(x(t)) \phi=0 .
$$

A direct application of Theorems 1 and 2 yields obviously

THEOREM 3. Let $\lambda_{n}, \mu_{n}, \Lambda_{n}$ be the eigenvalues of (4.2) with the boundary equations (4.3), (4.4), or (4.5) respectively. Under the assumptions

(i) $\sigma \rho(-x)=\sigma \rho(x)$,

(ii)' $\sigma \rho$ increasing in $(-1,0)$,

we then have $\lambda_{n}-2 \lambda_{1} \geq \mu_{n}$.

Under the assumption

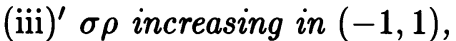
we then have $\Lambda_{n}-2 \Lambda_{1} \geq \mu_{n}$.

\section{REFERENCES}

1. R. Courant and D. Hilbert, Methods of mathematical physics, vol. 1, Interscience, New York, 1953.

2. L. E. Payne and H. F. Weinberger, An optimal Poincaré inequality for convex domains, Arch. Rational Mech. Anal. 5 (1960), 286-292.

3. B. Wang, S.-T. Yau and S. S.-T. Yau, An estimate of the gap of the first two eigenvalues in the Schrödinger operator, Ann. Scuola Norm. Sup. Pisa 12 (1985), 319-333.

MATHEMATISCHES INSTITUT, UNIVERSitÄt BASEL, CH-4051 BASEL, SWITZERLAND

DÉPARTEMENT DE MAThÉmatiques, Université LAVAL, QuÉbec, CANADA GIK 7P4 